\title{
Design of Electric Fire Monitoring System Based on Zig Bee
}

\author{
Biqing Li ${ }^{1}$, Suping Jiang 2 , a , Wenya Lai ${ }^{1, b, ~}{ }^{*}$, Yangming Chen ${ }^{1}$, Huanhua Huang ${ }^{1}$, \\ Huiliang Huang ${ }^{1}$, Shaohong Huang ${ }^{1}$, Yankui Zhao ${ }^{1}$, Liuren Wei ${ }^{1}$ \\ ${ }^{1}$ School of Information and Communication Engineering, Hezhou University, Hezhou Guangxi \\ 542899, China; \\ ${ }^{2}$ Guangxi Talent International College, Qinzhou Guangxi 535000, China. \\ a229292710@qq.com, b, ${ }^{*}$ janliful@163.com,
}

Keywords: CC2530ZigBee development kit; temperature and humidity sensor; smoke sensor.

\begin{abstract}
System is based on CC2530ZigBee development suite, is mainly composed of two parts, including the system power supply part, liquid crystal display part, buzzer alarm, temperature and humidity acquisition part, smoke collecting part, CC2530ZigBee development suite of terminal sections and the coordinator, etc. When the terminal part of the temperature and humidity sensors and smoke sensor is detected at the same time set temperature more than $35^{\circ} \mathrm{C}$ and smoke concentration exceeds bid, buzzer alarm, and through the Zig Bee wireless network transmit information to the coordinator to handle and displayed on the LCD when the value of temperature and humidity. Can reduce or eliminate losses of property in a timely manner. After the test, the design to achieve the desired effect.
\end{abstract}

\section{Overall Design}

\subsection{Design Thought.}

This design could be implemented according to the terminal temperature and humidity detector and smoke detector to collect environmental information by ZigBee wireless network transmission to the coordinator to display. When the protected circuit parameters over being detected in the alarm set value, it could send out alarm signals and control signals could indicate alarm parts of the system. It consisted of fire monitoring equipment (a device can receive signals from the alarm of fire monitor probe, making a sound and light alarm signals and control signals, indicating alarm parts, recording and saving the alarm information) and the monitoring and controlling of the fire detector (a detector is used to monitor the defense of fire risk parameters such as smoke, temperature in protected circuit).

\subsection{System Hardware Module.}

\subsubsection{Temperature and Humidity Sensor.}

In real life, environmental temperature and humidity was indispensable for people, which were closely related to people's life and production activities. Therefore, with the development of science and technology, temperature and humidity sensors would inevitably appear which had brought progress to people lives. Temperature and humidity sensor was referring to the constant temperature and humidity could be constant abstract shape into easy to measured processing of the electrical signal or digital signal, which was the real and touchable numbers, not just a concept of temperature and humidity. This topic selected the DTH11 series sensor, the physical appearance structure was shown in figure 1. 


\subsubsection{Introduction to Smoke Sensor.}

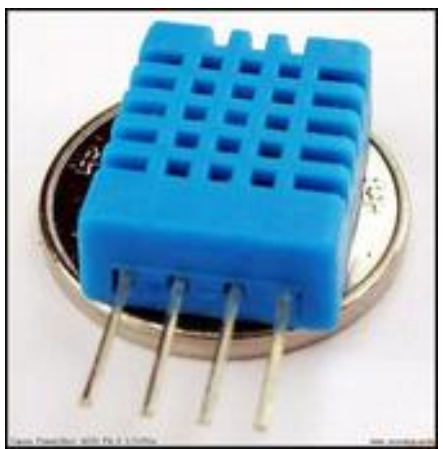

Figure 1. Humidity sensor DHT11

This topic selected the MQ-2 series sensor, and the physical appearance structure was shown in Figure. 2. This topic chosen ready-made module to connect the processor and adopted the module of digital output connection processor of the IO port. It by judging the high and low of electrical level of IO port judged the detected ready-made smoke scope whether reaches the standards of fire to trigger the alarm.

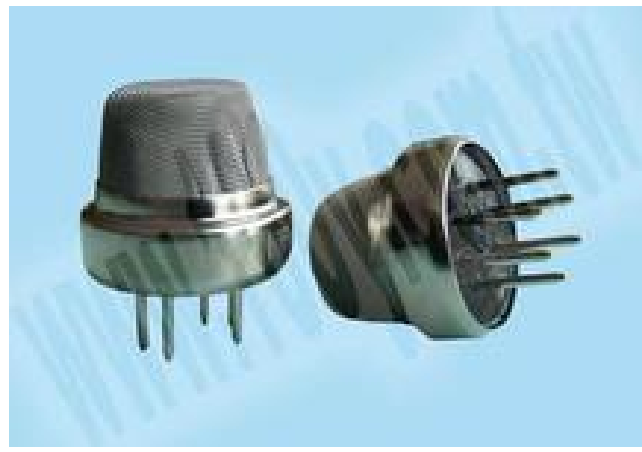

Figure 2. MQ-2 smoke sensor

\section{Hardware Circuit Design.}

According to the requirement of title of the first chapter and added functions, the total design principle mainly consisted of CC2530 ZigBee development suite circuit, smoke sampling circuit, temperature and humidity acquisition circuit, display circuit information, buzzer alarm circuit and power supply circuit. The circuit principle diagram respectively each part was as follows.

\subsection{Zig bee Core Circuit.}

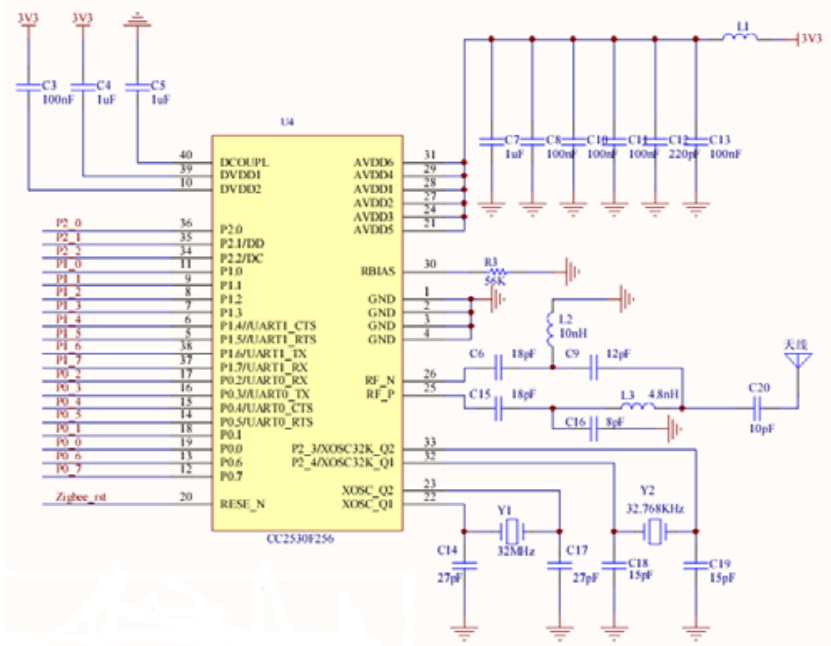

Figure 3. ZigBee core circuit

Zig Bee core circuit was shown in figure 7. The core circuit was divided into three parts, respectively the power part, the clock part and the radio frequency antenna unit. The following were detailed principles illustration for these three sections. 
(1) The power parts

The design of this part adopted external dry cell to supply power to the system, and the power supply voltage was $3.3 \mathrm{~V}$. The voltage of $3.3 \mathrm{~V}$ came from the output of the power supply circuit designed by the system. The specific situation was shown in the upper part of figure 7 . The power pins, such as, CC2530 DVDD1 and DVDD1, as well as AVDD1, AVDD2, AVDD3, AVDD4, AVDD5, AVDD6 needed to be connected to 3.3V power. CC2530's 40th pin was connected to GND.

(2) The clock parts

The chip of the clock part was composed of two different frequency parts, respectively $32 \mathrm{MHz}$ and $32.768 \mathrm{KHz}$. The $32 \mathrm{MHz}$ frequency supply was provided the system for normal working clock frequency, which was connected to the port P22 and P23 of CC2530 respectively. And $32.768 \mathrm{KHz}$ provided the operating frequency of the system in low-power mode to save energy. It was connected to the port P32 and P33 of CC2530 respectively.

\subsection{Circuit Design of Coordinator.}

The main circuit of the coordinator node includeed power supply circuit, ZigBee core circuit, ZigBee debugging circuit, and liquid crystal display circuit, power indicator light and communication indicator light. The circuit of the coordinator node was similar or most parts were like the circuit of the terminal node, and not all of them should have specific analysis. Here, only the liquid crystal display circuit and the communication indicator light were analyzed.

\subsubsection{LCD Display Circuit.}

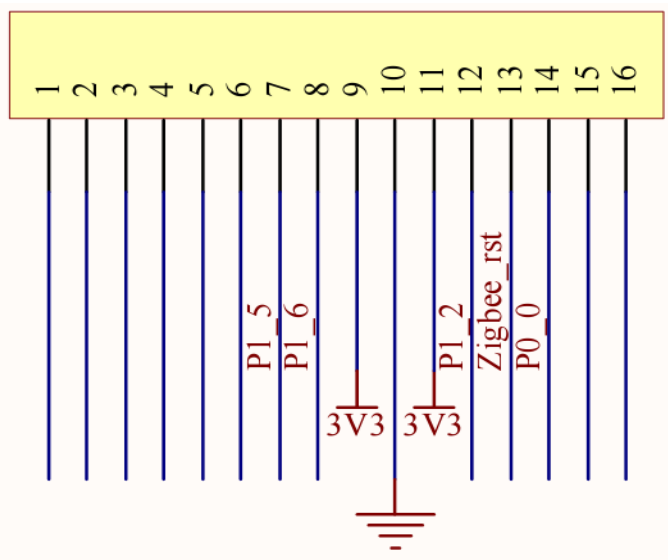

Figure 4.LCD circuit

The liquid crystal module adopted 12864 liquid crystal modules to save the pin of CC2530, adopted the serial method. The P00 pin, P12 pin, P15 pin and P16 pin of CC2530 were connected to the data port of the liquid crystal module. LCD module as an important module of the coordinator node mainly displayed information about the data sent from the terminal. This topic mainly displayed information of terminal node bed from the temperature and humidity and smoke scope. When real-time temperature and smoke exceed the temperature value set by the program, the receiving end 12864 would display real-time temperature information from the sending end to trigger the alarm circuit. Because of its high integrity, this greatly simplified hardware circuit, which meat it would increase the reliability of the system. It had long durable years and was not easy to damage. It worked under voltage of 5 volts, so it and CC2530 were power-sharing and easy to control.

\section{Debugging}

Debugging was mainly based on hardware design and programming to see if the final design achieved the desired results. In this design, according to the requirements of selected topic, it designed the hardware modules. And then to write modules programs according to the hardware, and the last to connect to the writing programs that the modules in the main function download the system operation to observe the effect.

\subsection{System Hardware Debugging.}

(1) Visual inspection to check whether the development board had rosin joints, or a joint flash. 
(2) Multimeter test to check whether there was a short circuit between various power lines and ground wires.

(3) Overall inspection to avoid generating useless parasitic capacitors, and when placing the capacitor, it was best to close to the SCM.

In this design, for convenient to change and carry, it adopted battery-power. Section 3 dry batteries installing in series, and then through the voltage transformation chip, it supplied power to the entire system. The experimental results showed that with the method of a battery-powered, it could make the whole system to work properly.

\subsection{Program Debugging.}

The debugging of software was mainly completed in the IAR integration environment. The function of the coordinator was relatively simple. It completed the transparent transmission of sensor module of collection sensor terminal note. The software design of the coordinator was mainly written in the application layer.

\section{Summary}

The paper is about the design of System is based on CC2530ZigBee development suite, is mainly composed of two parts, including the system power supply part, liquid crystal display part, buzzer alarm, temperature and humidity acquisition part, smoke collecting part, CC2530ZigBee development suite of terminal sections and the coordinator, etc.

\section{Acknowledgments}

This work is supported the following fund:

2017 The student work subject program of Huizhou University: "college students' network addiction of the student work service research" (No, hzxysz201707).

2016 The project of improving the basic ability of young teachers in Colleges and universities in Guangxi:"An empirical study of Guangxi Beau Gulf Economic Zone Foreign Trade Company foreign trade English correspondence" (No, KY2016YB886).

2016 The higher education research subject project of Huizhou University: "between collaborative innovation researches of entrepreneurial talent cultivation system".

2017 college students' innovative entrepreneurial project: "The agricultural robot autonomous operations research based on machine vision"(No,201711838096) \&\&"Multi-functional autonomous agriculture robot development"(No,201711838121) \&\&"Research and development of navigation system for agricultural robot"(No,201711838008) \&\&"Research and development of data acquisition system and servo control system for mobile platform of agricultural robot" (No,201711838075) \&\&"Research and development of visual navigation system for agricultural robot test platform"(No,201711838076).

2017 Guangxi education science 'ten-three-five' planning project: "Based on set's ICT education platform of fusion, work-integrated learning education training mode research" (No, 2017B107).

\section{References}

[1]. B.Q Li, Y.F Ling, H.Y Zhang, S. Y Zheng: The Design and Realization of Cherry Tomato Harvesting Robot Based on IOT. International Journal of Online Engineering, 12(12), 23, (2016).

[2]. B.Q Li, W.L Guan, S.Y Zheng, X.G Yue: Optimisation Design of Corn Precision Seder Based on Multi-Route and Multi-Channel Control. Journal of the Balkan Tribological Association, 21(4a), 1215, (2015). 
[3]. S.Y Zheng, et al, Social Work in Teen Addiction Correction Services Research under the New Situation, in: Proceedings of the 2015 4th National Conference on Electrical, Electronics and Computer Engineering (Niece 2015), Xian, China, 2015, Pp.252-255.

[4]. B.Q Li, et al, Intelligent Control Management System and Its Application, in: Proceedings of the 2016 International Conference on Economics and Management Innovations, Wuhan, China, 2016, Pp.68-71.

[5]. B.Q Li, et al, Design and Implementation of Tanks War Game Based on the Android Platform, in: Proceedings of the 2016 2nd Workshop on Advanced Research and Technology in Industry Applications, Dalian, China, 2016, Pp.963-966.

[6]. B.Q Li, et al, Design of a Tea Garden Ant freezing Control System, in: Proceedings of the 2016 6th International Conference on Machinery, Materials, Environment, Biotechnology and Computer (Mimic), Tianjin, China, 2016, pp.736-738.

[7]. B.Q Li, et al, Design of Electronic Compass, in: Proceedings of the 2016 6th International Conference on Machinery, Materials, Environment, Biotechnology and Computer (Mimic), Tianjin, China, 2016, Pp.1240-1243.

[8]. B.Q Li, Et Al, Research of Automatically Light-Adjusting Lamp, In: Proceedings of the 2016 International Conference on Computer Engineering, Information Science \& Application Technology (Icier 2016), Guilin, China, 2016, Pp.249-252.

[9]. B.Q Li, et al, The Design and Realization of Fruit Harvesting Robot Based on Iota, In: Proceedings of the 2016 International Conference on Computer Engineering, Information Science \& Application Technology (Ickier 2016), Guilin, China, 2016, PP.261-264.

[10]. B.Q Li, et al, A New Type of Automatic Opening and Closing Light-Operated Curtain, in: Proceedings of the 2016 International Conference on Mechatronics Engineering and Information Technology (Iceman), Xian, China, 2016, Pp.66-69.

[11]. B.Q Li, et al, Design of the Intelligent Air Humidifier, in: Proceedings of the 2016 International Conference on Mechatronics Engineering and Information Technology (Iceman), Xian, China, 2016, Pp.201-203.

[12]. B.Q Li, et al, The Design Implementation of the APP of Experiencing Guangxi Folk Custom, in: Proceedings of the 2016 International Conference on Economics and Management Innovations, Wuhan, China, 2016, Pp.47-50.

[13]. S.Y Zheng, et al, Digital Display Design of Ethnic Clothing of Nanking, in: Proceedings of the 4th International Conference on Mechatronics, arterials. Chemistry and Computer Engineering 2015(Icmmcce 2015), Xian, China, 2015, Pp.2805-2808.

[14]. S.Y Zheng, et al, Design and Implementation of Supermarket Personnel Management System Based on Java, in: Proceedings of the 2015 International Conference on Education, Management and Computing Technology, Tianjin, China, 2015, Pp.1724-1727.

[15]. S.Y Zheng, Et Al, Analysis of Internet of Things Talent Training and Curriculum System Innovation, In: Proceedings of the 2016 International Conference on Education, Management and Computing Technology(Icemct-16), Hangzhou, China, 2016, PP.957-960.

[16]. S.Y Zheng, et al, Brief analysis on "HeYuanTong" Campus Mobile Phone APP Design, in: Proceedings of the 2015 5th International Conference on Computer Sciences and Automation Engineering, Sonya, China, 2015, Pp.151-154. 